\title{
Quality control of cosmetics: case of baby wipes sold in Yaounde
}

\begin{abstract}
Cosmetic products have increasingly bad reputation because the risks associated to their uses unleashed passions and controversy; special attention is therefore required in the use especially those intended for children under 03 years, given the specific anatomical and physiological fragility of their skin. Among cosmetics for children, wipes are included in the search results because their use is convenient and does not require water. They are sold all over the world, specially designed to clean the baby's seat area. Our study consisted to determine the technico-regulatory, physicochemical and microbiological quality these baby wipes sold in the city of Yaounde. 07 groups of samples representing 07 brands of baby wipes most sold in Yaounde were analyzed according to the European Cosmetic Regulation EC No. 1223/2009 and the General Standard for cosmetics in Cameroon (NC 801: 2013). In addition, an inventory of the wipes frequency of use in Yaounde has been done, to guide us on the choice of brands to analyze. We founded as results that the non-conformity rate was $100 \%$ for the label control, $71.43 \%$ for physicochemical tests (with phenoxyethanol detected in $43 \%$ of samples tested and MIT in $28.57 \%$ of samples tested) and also $28.57 \%$ nonconformity rate for microbiological testing. At the end, an evaluation of these results has permit identified some causes of non-conformities such as non-compliance with standards rules, poor storage conditions in the retail outlets and the absence of a cosmetic regulations to Cameroon. To overcome these problems, recommendations were made intended to all stakeholders, including organizing awareness campaigns on babies cosmetic product safety and the importance of reading labels of cosmetics, establish a national strategy for systematic control of cosmetics quality and a redraw from the market all wipes containing prohibited ingredients such as Methylisothiazolinone, phenoxyethanol.
\end{abstract}

Volume 7 Issue 2 - 2019

\author{
Rostand Sagu Kenfack, ${ }^{1,2}$ Rose Ngono \\ Mballa, ${ }^{1,4}$ Marcel Evrard Nguidjoe, ${ }^{3}$ Pernel \\ Libam IV,' Logmo Mahi, ${ }^{4}$ Edmond Tsafack, ${ }^{3}$ \\ Aminatou Sarbe Manon, ${ }^{6}$ Philippe Piccerelle ${ }^{5}$ \\ 'National Drugs Quality Control and Valuation Laboratory, \\ Cameroon \\ ${ }^{2}$ CRESA-BOIS Nkolbisson, University of Dschang, Cameroon \\ ${ }^{3}$ Department of Pharmacotoxicology and Pharmacokinetics, \\ University of Yaounde I, Cameroon \\ ${ }^{4}$ Department of Pharmacology and Traditional Medicine, \\ University of Yaounde I, Cameroon \\ ${ }^{5}$ Laboratory of Pharmaceutics Industrial, Cosmetology and \\ Bioengineering Pharmaceutical, Faculty of Pharmacy, University \\ Mediterranean, France \\ ${ }^{6}$ Visatox, France
}

Correspondence: Rose Ngono Mballa, Department of Pharmacology and Traditional Medicine, University of Yaounde I, Cameroon, Email princessrose@hotmail.fr

Received: March 27, 2019 | Published: April 23, 2019

Keywords: quality control, cosmetics, cosmetic ingredients, safety

\section{Introduction}

According to the Cosmetics Regulation (EC) No 1223/2009, a cosmetic is "any substance or preparation intended to be placed in contact with the various external parts of the body including the skin, hair and capillary systems, nails, lips and external genital organs or with the teeth and the oral mucosa, with an aim exclusively or mainly to cleaning them, perfuming them, changing their appearance, protect, maintain in good condition or correcting body odors." This regulation does not foresee any authorization for the placing on the market of cosmetic products, but requires those responsible for placing on the market to ensure the safety of these products. Each cosmetic product is considered to be the result of a combination of ingredients. ${ }^{1}$ Therefore, the regulation (EC) No 1223/2009 imposes an assessment of ingredients including: the absence of prohibited substances, the restrictions, compliance labeling, good microbiological quality and conformity of physicochemical analysis $(\mathrm{pH}$, allergens, preservatives), especially for products intended to children under 03 years. According to the European classification of cosmetics, baby wipes belong to first-class cosmetic products and are non-rinsed. ${ }^{2}$ These products are generally composed of a non-woven substrate made of synthetic fibers and/or cellulose, impregnated with a cleaning substance which some manufacturers sayit contains parabens, phenoxyethanol, isothiazolinones, etc. ${ }^{3}$ This complex composition is likely to present risks to public health in contact with the delicate skin of young children. This study to evaluate the microbiological quality of outstanding wipes in Yaounde and their physicochemical composition in order to communicate the risks associated with use of these cosmetic.

\section{Materials and methods}

\section{Materials used for data collection}

A preliminary investigation concerning the use of baby wipes was conducted in Yaounde over a period of 21 days with a guide sheet consists of 13 questions. The survey covered 450 individuals considered as key item regarding the health of children, including 400 women from all social classes and 50 health professionals.

\section{Control of labeling}

The conformity assessment of the information mentioned on the label wipes marketed was done according to the requirements of the Cameroon CN standard 804: 2013, published by the Agency for Standards and Quality (ANOR).

\section{pH measurement}

The $\mathrm{pH}$ measurement was performed using qualify and calibrated $\mathrm{pH}$ meter. For each sample tested, 03 reading $\mathrm{pH}$ values were taken to determine the mean. For this test, we relied on the recommendations of the National Agency of Medicines Safety and Health products (ANSM) in 2012 about the safety of products intended for children under 03 years. ${ }^{4}$ Thus, a wipe will be considered safe for the child's health if it has a $\mathrm{pH}$ value between 4.5 and 6 .

\section{Highlighting alcohols present}

Highlighting alcohols present was made by a color test with potassium dichromate, a method referenced in the collection. ${ }^{5}$ For this analysis, we extracted gently in a beaker of $10 \mathrm{ml}, 5 \mathrm{ml}$ of the 
wipes moisturizing lotion and $2 \mathrm{ml}$ of the initially prepared solution of potassium dichromate was add to observe the change of color.

\section{Search of cosmetic prohibited ingredients in wipes}

\section{Phenoxyethanol or 2-phenoxyethanol:}

A preliminary extraction was made in distilled water at $37^{\circ} \mathrm{C}$ and the identification was done by UV-visible spectrophotometry (according to European Pharmacopoeia ${ }^{6}$ ) with a reading spectral region from 240 to $350 \mathrm{~nm}$. Phenoxyethanol absorption spectrum shows two peaks with absorption maximum wavelength at $269 \mathrm{~nm}$ and $275 \mathrm{~nm}$.

The Methylisothiazolinone (MIT) or 2-Methyl-4-isothiazolin3-one:

A preliminary extraction was made in ethanol $95^{\circ} \mathrm{C}$ and the resulting mixture placed in an ultrasound bath for $30 \mathrm{~min}$ at $25^{\circ} \mathrm{C}$. The identification was done by UV-visible spectrophotometry using a method described, ${ }^{7}$ with the reading of a single spectrum maximum absorption at $275 \mathrm{~nm}$. The calculation of concentrations was made following Beer Lambert law and calculation of the MIT safety margin was based on its systemic exposure and toxicological parameters. According to the guidelines of the SCCS (Scientific Committee on Consumer Safety) in 2012, ${ }^{8}$ these safety margin calculations are performed using the following formula.

\section{Microbiological analysis}

The volume taken for analysis was $5 \mathrm{ml}$ for each trial ( 5 trials per sample) and diluted to one-tenth $(1 / 10)$. We focused our samples by vacuum filtration. To evaluate the microbiological conformity, three tests were carried out in particular, a total yeasts and molds count on TSA agar (ISO/TS $11133-2 / \mathrm{A} 1$ ) incubated at $30^{\circ} \mathrm{C}$ for 5 days (and validation of enumeration on SDA: Sabouraud dextrose agar for Candida albicans); enumeration and validation of total mesophilic aerobic organisms on PCA agar (ISO 4833) incubated at $37^{\circ} \mathrm{C}$ for 72 $\mathrm{h}$ (in search of Staphylococcus aureus and Pseudomonas aeruginosa), finally a specific search for Pseudomonas aeruginosa on Mueller Hinton agar incubate the medium at $37^{\circ} \mathrm{C}$ for $48 \mathrm{~h}$. Given the origin of different brands of wipes, analysis of our results was done according to the European Cosmetic Regulation EC N ${ }^{\circ} 1223 / 2009$ and to the General Standard for cosmetics in Cameroon (NC 801: 2013). ${ }^{9}$

\section{Results}

\section{Results from survey forms}

Brands of baby wipes used in yaounde: 400 women were interviewed concerning the use baby wipes: 338 women (84.5\%) used wipes for hygiene of their children and 67 women $(15.5 \%)$ do not used wipes for their children; What has therefore helped to identify 20 brands of baby wipes used in Yaounde but only 07 brands have retained our attention due to their high percentages in terms of use.

Public awareness level on the risk of babies wipes toxicity: Table 1 showed that majority of the tested population $(84.89 \%)$ was not aware of the risk of baby wipes toxicity, including some health professionals.

Distribution of samples for analysis: Table 2

\section{Labeling control}

One hundred percent (100\%) of the brands controlled was in nonconformity regarding the labeling. The main discrepancies were: the absence of mandatory particulars, misuse of language, lack of batch number and an incomplete list of ingredients.

Table I Public awareness levels on the risk of babies wipes toxicity

\begin{tabular}{llll}
\hline Sensitization & & Workforce & Percentage \\
\hline \multirow{2}{*}{ Mother } & YES & 36 & YES $=15.11 \%$ \\
& NO & 364 & \\
Health professional & YES & 32 & NO $=84.89 \%$ \\
& NO & 18 & \\
Total & & 450 & $100 \%$ \\
\hline
\end{tabular}

YES, people already aware of the toxicity of baby wipes.

$\mathrm{NO}$, people not aware of the toxicity of baby wipes.

\section{Physico-chemical analysis}

pH measurement: Figure 1 shows that

a. The $\mathrm{pH}$ values are different depending on the samples, but all oscillate in the range of the recommended $\mathrm{pH}$ for children, with the exception of E6 and E7 coming out of the standard;

b. The measured $\mathrm{pH}$ does not match the $\mathrm{pH}$ indicated on the labels. Amount the 07 samples tested, 02 were indications of the $\mathrm{pH}$ (5.5), it is revealed during the testing of different $\mathrm{pH}$ values of that found on the labels.

Highlighting of alcohols presence in wipes: This test revealed the presence of alcohol in $28.57 \%$ of samples.
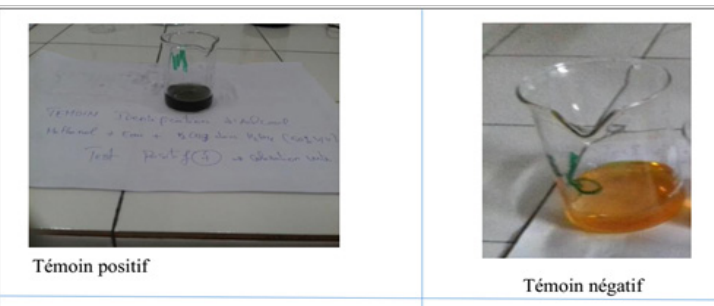

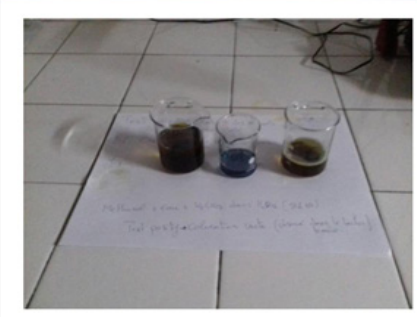

Tests positifs à l'alcool

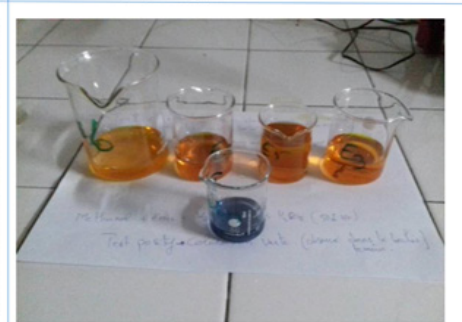

Tests négatif à l'alcool
Photo 1 Colorful test with Potassium dichromate

Identification of Phenoxyethanol: For the 07 samples tested, 03 $(42.86 \%)$ showed positive results with each presenting two peaks with maximum absorption around $269 \mathrm{~nm}$ and $275 \mathrm{~nm}$; these include E1, E2, and E7. The other 04 samples $(57.14 \%)$ had peaks which were however not specific to those of phenoxyethanol (Figure 2).

\section{Identification and dosage of Methylisothiazolinone (MIT)}

UV spectra revealed that amount the 07 samples tested, 02 (28.57\%) had a maximum absorption peak around $275 \mathrm{~nm}$ specific to MIT, with different absorbance (Figure 3). 
Table 2 Samples of wipes collected for analysis

\begin{tabular}{llllllc}
\hline Brands & Baby Wipes & Moby Baby & $\begin{array}{l}\text { Fresh } \\
\text { Runy }\end{array}$ & Baby Sita & Oridel & $\begin{array}{l}\text { Corine De } \\
\text { Farne }\end{array}$ \\
\hline Quantity & 3 & 3 & 3 & 3 & 3 & 3 \\
$\begin{array}{l}\text { Collection } \\
\text { site }\end{array}$ & $\begin{array}{l}\text { Ngoa-Ekele } \\
\text { perfumery }\end{array}$ & $\begin{array}{l}\text { Santa Lucia } \\
\text { Mokolo }\end{array}$ & $\begin{array}{l}\text { DovV } \\
\text { Mokolo }\end{array}$ & $\begin{array}{l}\text { Central Market } \\
\text { perfumery }\end{array}$ & $\begin{array}{l}\text { Omnisport } \\
\text { perfumery }\end{array}$ & $\begin{array}{l}\text { Casino } \\
\text { supermarket }\end{array}$ \\
\hline
\end{tabular}

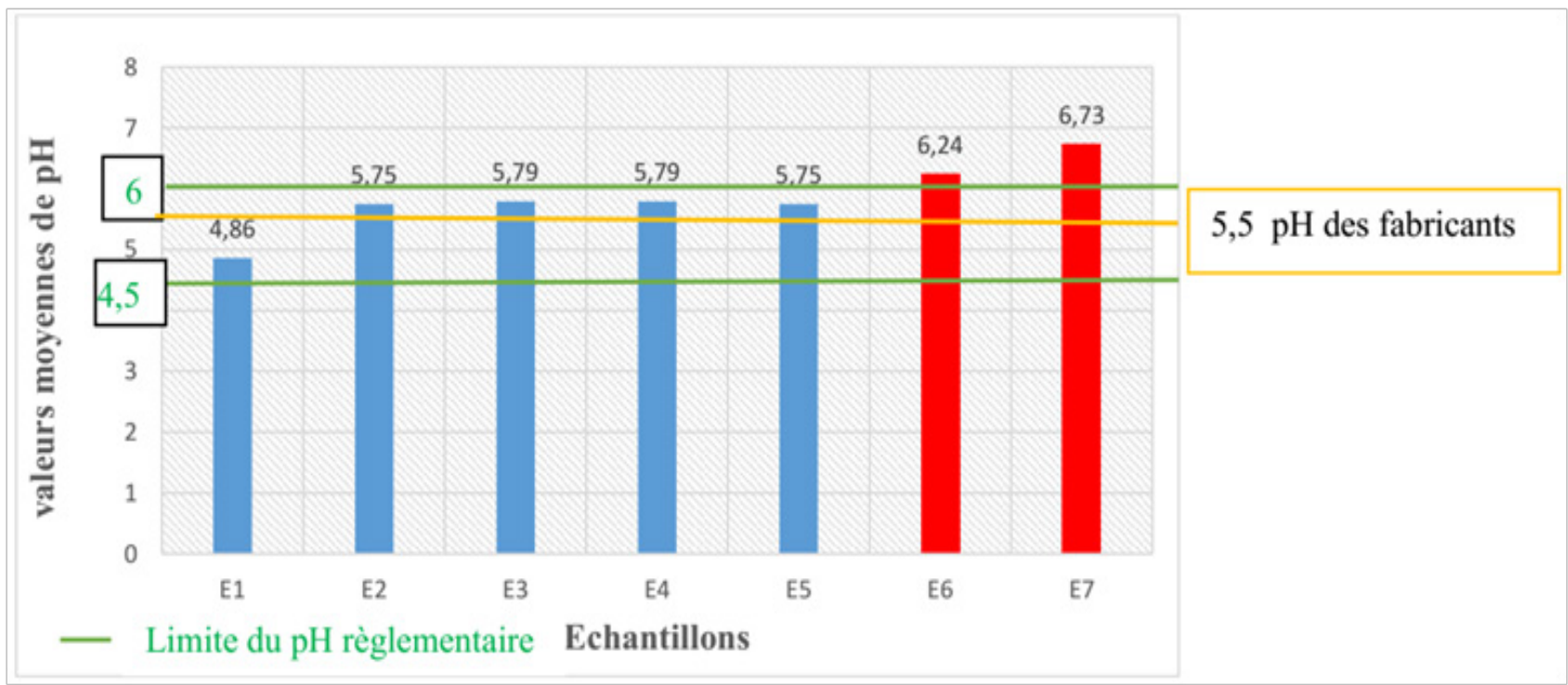

Figure I pH average values compared to the norm recommendation.

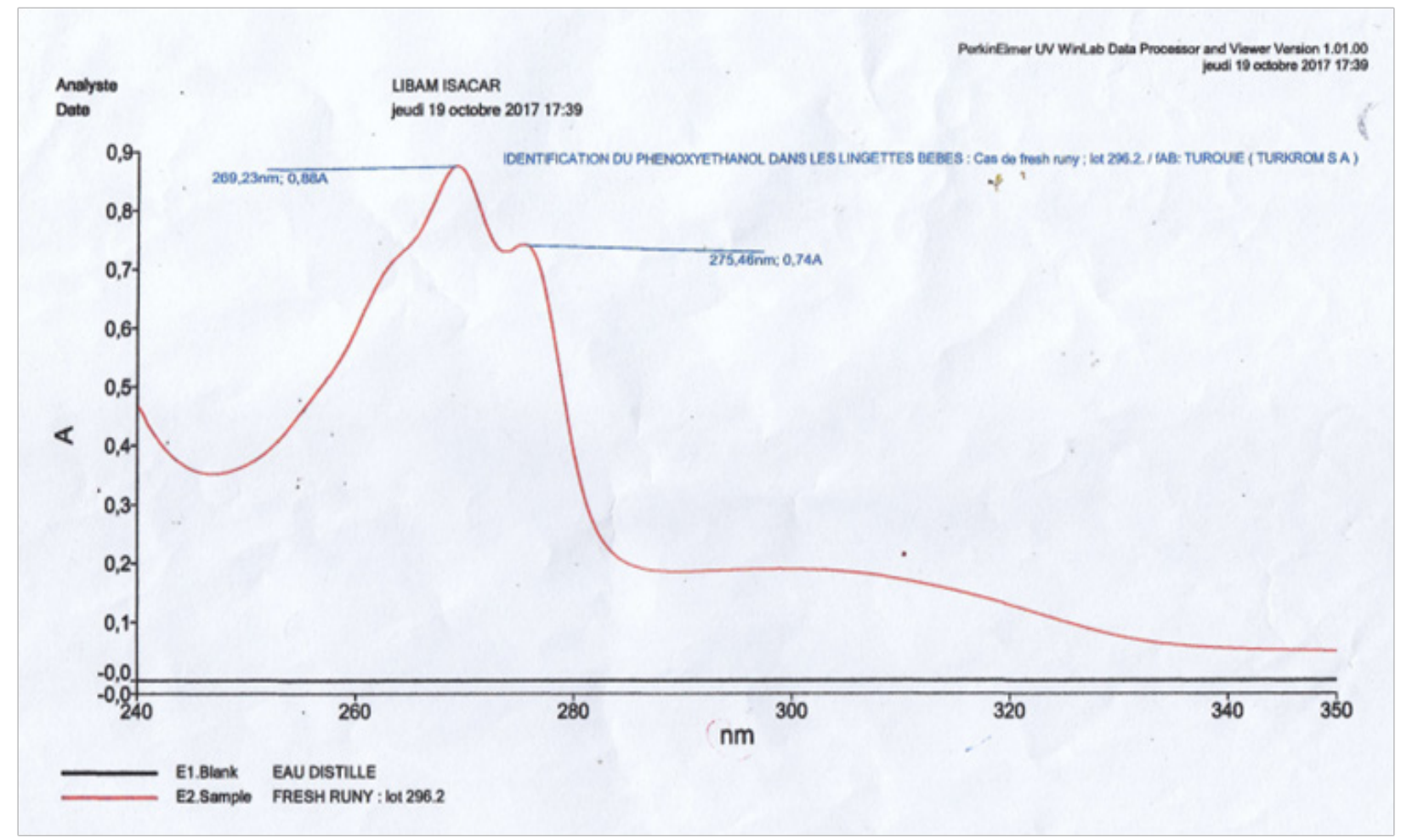

Figure 2 Some identification results of phenoxyethanol on UV spectrophotometer. 


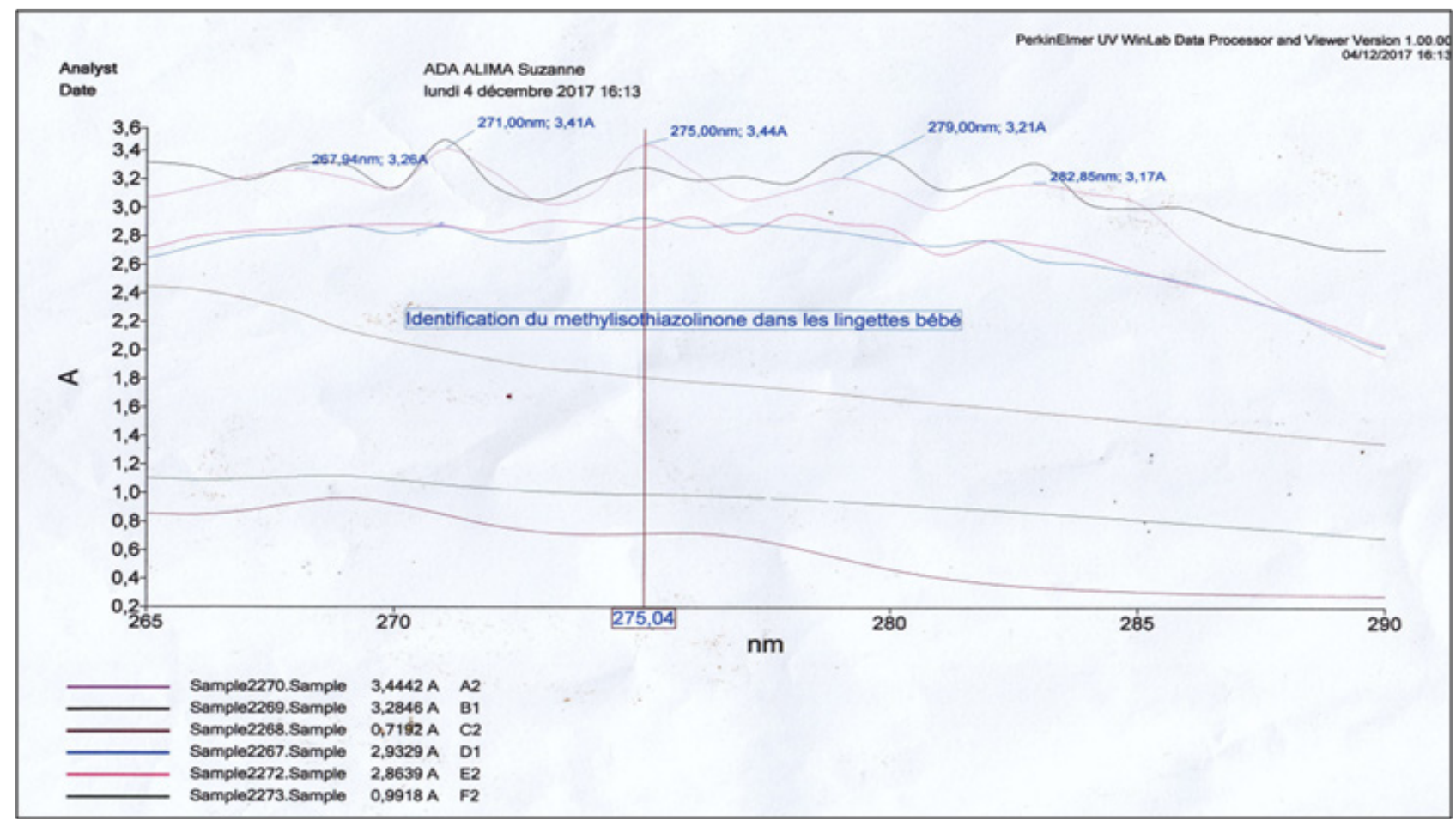

Figure 3 Some UV spectra for different MIT samples tested.

For each positive case, we determined the concentration of MIT (ppm) present in the wipes and calculated the safety margins (MoS) for each sample. The set results of the MoS are shown in Table 3.

Given all this values, the following can be outlined:

a. The frequency use chosen ( $\mathrm{F}=3,6$ and 15 wipes/day) cover the frequencies obtained during the investigation. Indeed the manufacturer did not indicate on the packaging the number of wipes to use daily. This variability in calculations allows us to determine the acceptable limit of daily use. b. Only3 wipes/day of E1 has an acceptable safety margin (MoS): $10342>100$ (the standard being strictly greater).

c. For sample E5, at $\mathrm{F}=3,6$ and 15 wipes/day, the $\mathrm{MoS}$ is not acceptable, lower than the standard. This means that even within 3 wipes/day for E5, children remain at high risk of toxicity from MIT.

d. MIT safety margin in wipes is unacceptable.

Table 3 Summary table of the MoS evaluation

\begin{tabular}{|c|c|c|c|c|c|c|}
\hline Samples & \multicolumn{3}{|l|}{ EI } & \multicolumn{3}{|l|}{ E2 } \\
\hline MMIT total (g) & \multicolumn{3}{|l|}{0.000644} & \multicolumn{3}{|c|}{0.0000704} \\
\hline Number of wipes & \multicolumn{3}{|l|}{10} & \multicolumn{3}{|l|}{7} \\
\hline MIT m per wipe (g) & \multicolumn{3}{|l|}{0.0000644} & \multicolumn{3}{|c|}{0.0001006} \\
\hline NOAEL (Mg/kg bw/day) & \multicolumn{3}{|l|}{2} & \multicolumn{3}{|l|}{2} \\
\hline Frequency of application day $=\mathrm{F}$ & 3 & 6 & 15 & 3 & 6 & 15 \\
\hline SED (Mg/kg/day) & 0.019338 & 0.038676 & 0,09669 & 0.0302 & 0.0604 & 0,151 \\
\hline$M o S>100$ & 103,42 & 51,71 & 20,68 & 66.225 & 33.1125 & 13.26 \\
\hline
\end{tabular}

\section{Microbiological analysis}

The identified microbiological conformity concerned $92.96 \%$ of the samples on TSA, $92.96 \%$ of the samples on PCA and $100 \%$ sample on Mueller Hinton agar. Regarding the microbiological stability, the compliance rate was $85.71 \%$. The level of microorganisms present in the baby wipes was $7.04 \%$ for the mesophilic aerobic germs, referring to Staphylococcus aureus (black and shiny colonies, Gram + shell, Catalase + , Coagulase + ) after validation of the detection on Baird 
parker agar and total absence of Pseudomonas aeruginosa. We also noted the presence of yeasts and molds at a rate of $7.04 \%$, referring to Candida albicans (convex and creamy colonies, beige color) after validation of detection on SDA agar.

\section{Summary of results obtained}

For all tests, the Table 4 below provides a summary of results obtained in terms of conformities.

Table 4 Summary of overall compliance based on the results

\begin{tabular}{|c|c|c|c|c|c|c|}
\hline Brands & Control of labeling & \multicolumn{4}{|c|}{ Physicochemical control } & \multirow[t]{2}{*}{$\begin{array}{l}\text { Microbiological } \\
\text { control }\end{array}$} \\
\hline & & $\mathrm{pH}$ & Alcohol & $\begin{array}{l}\text { Phenoxy } \\
\text { ethanol }\end{array}$ & MIT & \\
\hline EI & $\mathrm{NC}$ & C & $\mathrm{NC}$ & $\mathrm{NC}$ & $\mathrm{NC}$ & C \\
\hline E2 & $\mathrm{NC}$ & C & C & $\mathrm{NC}$ & C & C \\
\hline E3 & NC & C & C & C & C & $\mathrm{NC}$ \\
\hline E4 & $\mathrm{NC}$ & C & NC & C & C & C \\
\hline E5 & NC & C & C & C & NC & $\mathrm{NC}$ \\
\hline E6 & NC & NC & C & C & C & C \\
\hline E7 & $\mathrm{NC}$ & NC & C & $\mathrm{NC}$ & C & C \\
\hline
\end{tabular}

C, confirm NC, Not confirm

\section{Discussion of results}

The results of our survey showed that more than 8 in $10(84.89 \%$ of the surveyed population) has never thought or even heard of wipes toxicity for their children. These results highlight a real need for public information. Indeed, if the small majority of the population (68 people out of 400 , or $15.11 \%$ ) has heard of the toxicity of baby wipes, knowledge remains generally sketchy and the information received is perceived as unsatisfactory for the most part. That ignorance of each other and this uncertainty is related to the poor communication and lack of awareness from healthcare organizations, added to the complexity of the product, as well they specified the Morocco Anti Poison Centers in its report in 2010. ${ }^{10}$

The labeling control result (100\% of non-compliance) differs from that of the DGCCRF (Directorate General for Competition, Consumer Affairs and Fraud Control) in France, in 2011, which carried out checks on close to 8000 cosmetics, and detected over 600 cosmetic with a non-compliant labeling representing $7.5 \%$ of non-compliance of labeling. The information present on the label, contributes to traceability and product safety use. This allows us to say that any lack of essential information about the wipes label, reflects negligence on the part of manufacturers, as emphasized by ANSM in France ${ }^{2}$ in its report on the safety of cosmetic products for children less than 03 years. Finally, the controls showed an increase of type "Free" claims ("allergen-free", "paraben free", "chlorine free") some, incorrect or unfair allegations, likely to disturb the perception of the consumer. It is the same for highlighted ingredients "noble" as they appear in the composition sometimes extremely low rates.

For the 07 samples analyzed, 05 had a $\mathrm{pH}$ value located in the range of references ( 4.5 and 6 ), that is $71.43 \%$ of conformity against $28.57 \%$ Non-conformity. Note also that these measured $\mathrm{pH}$ values did not corresponded to those indicated on the label wipes (5.5). This result can be explained either by poor product quality (misrepresentation by the manufacturer on the $\mathrm{pH}$ of the final product) or by poor storage conditions in the retail outlets. According to the French Observatory of Cosmetics (OFC) in February 2018, ${ }^{11}$ the $\mathrm{pH}$ values may decrease or increase depending on storage conditions such as temperature, which is generally not specified by the manufacturer and even less respected by saling points. The test with Potassium dichromate is a method included in the European Pharmacopoeia 2008 for the detection of alcohol in products. For recorded positive tests $(28.57 \%)$, one could read on about $50 \%$ of the samples the presence of alcohol. For the other $(50 \%)$, the presence of alcohol has not been mentioned. This situation is even more serious when they notice that it's written on the positive samples controlled "alcohol free".

Of the 07 samples examined, phenoxyethanol was detected in 03 samples (43\%) and MIT in 02 samples (28.57\% of the samples tested). These differ from those of the NGOs WECF published in 2016 after an investigation in France over 299 cosmetic products for babies. ${ }^{12}$ They founded that the Methylisothiazolinone appeared in 19 products $(6.3 \%$ of the tested products) and phenoxyethanol in 54 products $(18.06 \%$ of the products tested). Similarly, French Consumers Union (UFC) in $2017^{13}$ had detected 23 products out of $1000(2.3 \%)$ containing these two substances. It is also important to note that 01 of the 07 products (14.28\%) contained both phenoxyethanol and MIT. Phenoxyethanol appear in the list of prohibited substances (1328 substances) or negative list (Annex II) of Cosmetic Regulation (EC) No 1223/2009.14 The dosage and the calculation of the MIT safety margin for the wipes were made when this ingredient still belonged to the list of substances subject to restriction (Annex III) of the cosmetics regulation, but since 27 January 2018 the MIT is a prohibited substance in cosmetics. ${ }^{11}$

The microbiological compliance concerned $92.96 \%$ of the samples on TSA (meaning only $7.04 \%$ of total yeasts and molds count on TSA), $92.96 \%$ of the samples on PCA (meaning only $7.04 \%$ total aerobic mesophilic germ counts on PCA agar) and $100 \%$ sample on Mueller Hinton agar (absence of Pseudomonas aeruginosa). These results reflect the fact that manufacturers of $71.43 \%$ of tested samples met the good manufacturing practices (ISO 22716).Detected pathogenic microorganisms such as Staphylococcus aureus and Candida albicans are present small quantity, therefore complies with the standard ( $\leq$ $102 \mathrm{CFU} / \mathrm{ml}$ ), but this number could increase if the storage conditions are not respected. As for the microbiological stability, the results 
reflect the effectiveness of conservative utilities in $85.71 \%$ of the samples tested. As mentioned by some manufacturers, cosmetics and especially those who are used for a relatively long period, have an environment (temperature, $\mathrm{pH}$ and water) favorable to the development of microorganisms. ${ }^{15}$ (ISO/TS 11133-2/A1) incubated at $30^{\circ} \mathrm{C}$ for 5 days, the total aerobic mesophilic germ counts PCA agar (ISO 4833) incubated at $37^{\circ} \mathrm{C}$ for $72 \mathrm{~h}$ and Pseudomonas aeruginosa specific research on Mueller Hinton agar incubated at $37^{\circ} \mathrm{C}$ for 48 h. ${ }^{16-20}$

\section{Conclusion}

In conclusion we can say that children's skin is fragile in structure and the seat area is an occlusive environment with significant application frequency of wipes (about 06 times per day). The non-compliance rate was $100 \%$ for labeling control, $71.43 \%$ for physicochemical tests with the presence of two forbidden substance detected namely phenoxyethanol (hepatotoxic, reproductive toxicity, hematotoxic) and Methylisothiazolinone (a contact allergen, the main cause of skin irritation in children), and $28.57 \%$ of non-compliance for microbiological testing. These non-conformities expose children to serious health problems including: a bacterial infection, allergies, skin irritations, physiological disturbances, aggravated by the presence of faces and urine. Faced with this worrying situation, it is mandatory that the competent authorities to focus on assessing the quality and safety of cosmetic products for children control the import of cosmetics, cosmetovigilance and are developing a national strategy for systematic control of cosmetic products in circulation in Cameroon and mass awareness about precautions to take regarding the use of these products.

\section{Acknowledgments}

We first want to thank God Almighty, who gave us the strength, health and patience to accomplish this modest work.

Subsequently, we thank:

a. Dr NGONO MBALLA Rose, lecturer at Faculty of Medicine and Biomedical Sciences of the University of Yaounde I, for having accepted to supervise this work. We are deeply grateful for her interest and the time she gave us, for all the advice, support and encouragement throughout this work.

b. Dr NGUIDJOE Marcel Evrard, lecturer at Faculty of Medicine and Biomedical Sciences of the University of Yaounde I, for agreeing to co-supervise the realization of this modest work and his encouragement.

c. Mr. Ndjana Mvondo, our technical coach for his dedication and good explanations.

d. Dr AMINATOU Sarbe Manon, founder and manager of the company VisaTox, for his interest and the time she gave us, for all the advice, support and encouragement throughout this work.

e. Dr Logmo and MAHI, Dr Edmond Tsafack, for their time and for their strong support.

f. Dr Theophilus Kamgaing, Lecturer at the University of Dschang and Vice Coordinator CRESA, Responsible of Valuation and Insurance Control of medicines and Food Quality (CAQ) department, for his advices and assistance throughout the period of our studies. g. All LANACOME staff, for their cooperation, especially Mr. LIBAM IV Pernel Mr. Serge NDI Messi for their warm welcome, advice and support during our internship.

\section{Conflicts of interest}

Authors declare that there is no conflict of interest.

\section{References}

1. Laure C. Evolutions of the regulation of Cosmetics and impacts assessment of the safety for human health. Doctoral thesis presented and defended at the University of Caen; 2011. 155 p.

2. Risk assessment related to the use of phenoxyethanol in cosmetic products intended for children under 03 years. ANSM; 2012.

3. Complete results of the test carried out on 27 and 7 wipes cleansing milks. UFC Que Choisir No. 519; 2013.

4. Kongolo M. Issues and consequence of the use of cosmetics. Democratic Republic of the Congo: University of Lubumbashi; 2012. 80 p.

5. Clarke's analysis of drugs and poison. 3rd ed. Edited by Moffat AC, Osselton MD \& Widdop B, volume 1. Pharmaceutical Press; 2004. 648 p.

6. European Pharmacopoeia. 6th ed, Volume 2. France: Council of Europe; 2008. 3308 p.

7. Baranowska I, Wojciechowska I, Solarz N, et al. Determination of Preservatives in Cosmetics, Cleaning Agents and Pharmaceuticals Using Fast Liquid Chromatography. Journal of Chromatographic Science. 2014;52(1):88-94.

8. The SCCS'S Notes of Guidance for the testing of cosmetic ingredients and their safety assessment. 8th revision. SCCS/1501/12. CSAS; 2012. $133 \mathrm{p}$.

9. Castanedo-Tardana MP, Zug KA. Methylisothiazolinone. Dermatitis. 2013;24(1):2-6.

10. Recommendations for specific characteristics to be considered in assessing the safety of cosmetic products for children under 03 years. ANSM; 2010. 55 p.

11. Manon Aminatou Saber. Visatox. Loire Valley: France; 2018.

12. Annual Activity Report of the Agency on cosmetics. ANSM; 2016.

13. Undesirable substances in cosmetics over 1000 products pinned. UFC Que Choisir; 2017.

14. Regulation (EC) No. $1223 / 2009$ of the European Parliament and of the Council of 30/11/2009 on cosmetic products. Official Journal of the European Union; 2009. 151 p.

15. Control of cosmetic products on French territory. DGCCRF; 2016.

16. Directive 76/768/EEC of 27 July 1976 on the approximation of the laws of the States Member relating to cosmetic products. EUR-Lex; 1976. $169-200 \mathrm{p}$

17. Audouard M, Aulois-Griot M. Cosmetics products "borders": in search of a legal framework. Bull Pharm Order. 2004;385:591-599.

18. Beguin A. Diaper rash: still current. Arch Pediatric. 2006;13(3 suppl).

19. Zohra M. Safety assessment of cosmetic ingredients intended for children under 3 years. France: University of Bordeaux; 2014. 176 p.

20. Scheuplein R, Charnley G, Dourson M. Differential sensitivity of children and adults to chemical toxicity. I. Biological basis. Regul Toxicol Pharmacol. 2002;35(3):429-447. 